\title{
Histogram Equalization Based Enhancement and MR Brain Image Skull Stripping using Mathematical Morphology
}

\author{
Zahid Ullah ${ }^{1}$ and Su Lee ${ }^{1}$ \\ ${ }^{1}$ Changwon National University
}

May 5, 2020

\begin{abstract}
In brain image processing applications the skull stripping is an essential part to explore. In numerous medical image applications the skull stripping stage act as a pre-processing step as due to this stage the accuracy of diagnosis increases in the manifold. The MR image skull stripping stage removes the non-brain tissues from the brain part such as dura, skull, and scalp. Nowadays MRI is an emerging method for brain imaging. However, the existence of the skull region in the MR brain image and the low contrast are the two main drawbacks of magnetic resonance imaging. Therefore, we have proposed a method for contrast enhancement of brain MRI using histogram equalization techniques. While morphological image processing technique is used for skull stripping from MR brain image. We have implemented our proposed methodology in MATLAB R2015a platform. Mean square error (MSE) and peak signal to noise ratio (PSNR) has been used to evaluate the results of our presented method. The experimental results illustrate that our proposed method effectively enhance the image and remove the skull from brain MRI.
\end{abstract}

\section{Hosted file}

Enh_S_Stripping_.doc available at https://authorea.com/users/293356/articles/421310histogram-equalization-based-enhancement-and-mr-brain-image-skull-stripping-usingmathematical-morphology 